\title{
Editorial: Physics and Geomorphology of Sand Ripples on Earth and in the Solar System
}

\author{
Hezi Yizhaq ${ }^{1 *}$, Simone Silvestro ${ }^{2}$ and Klaus Kroy ${ }^{3}$ \\ ${ }^{1}$ Department of Solar Energy and Environmental Physics, Blaustein Institutes for Desert Research, Ben-Gurion University of the \\ Negev, Beersheba, Israel, ${ }^{2}$ Istituto Nazionale di Astrofisica, Osservatorio di Capodimonte, Napoli, Italy, ${ }^{3}$ Institute for Theoretical \\ Physics, Leipzig University, Leipzig, Germany
}

Keywords: impact ripples, large ripples, TARs, megaripples, sorting, reptation, wind drag ripples, reptation dunes

Editorial on the Research Topic

Physics and Geomorphology of Sand Ripples on Earth and in the Solar System

\section{IMPACT RIPPLES AND MEGARIPPLES}

Wavy sand patterns shaped by atmospheric flow, so-called aeolian bedforms, are abundant in arid regions on Earth, on the surface of Mars, and also on the surface of Venus and Titan (Day and Zimbelman, 2021; Tilman, 2021). In particular, aeolian impact ripples develop due to the instability of an initially flat bed of cohesion-less sand that is mobilized into a hopping motion called saltation (Figure 1A). These very common ripples emerge on relatively fine desert sands with typically unimodal grain size distributions and form rather parallel waves. In other words, they can be characterized as effectively two-dimensional bedforms, displaying only small crest modulations transverse to the wind direction. In contrast, so-called megaripples are more extreme forms that develop from poorly sorted sands and their crests exhibit greater sinuosity that increases with age (Yizhaq et al., 2019; Figure 1B). Interestingly, they are also characterized by bimodal grain-size distributions, with the coarser grains covering the crests and the finer fraction the troughs. The transport of the coarser grains occurs via a type of creeping or "reptation" mode, a succession of incremental grain movements. They are generally thought to be excited by impacts of the finer saltating grains (Figure 1B). Based on this observation, it has been suggested that megaripples can be mechanistically understood in close analogy to dunes. Namely, as an unconventional type of small dune (a so-called "reptation dune"), not merely decorated but rather created by coarse grains that are too heavy to participate in saltation but move in tiny steps when kicked by finer saltating grains (Lämmel et al., 2018). Interestingly, it was recently shown that megaripples can be active under the present climatic conditions on Mars, at least when associated with high sand flux dunes, indicating that past climate with a denser atmosphere may not be mandatory to explain their accumulation and migration (Silvestro et al., 2020). Sand transport under current Martian environment should actually even be facilitated and enhanced by the presence of polydisperse sand (Fu, 2020).

To date, the mechanism that saturates the growth of the impact ripples is one of the conundrums that are still not fully understood. A new DEM (Discrete Element Method) mathematical model for sand-ripple formation shows the importance of the grain size distribution in stabilizing the ripples growth (Huo et al., in this special issue). With monodisperse grains the ripple growth decreased in 


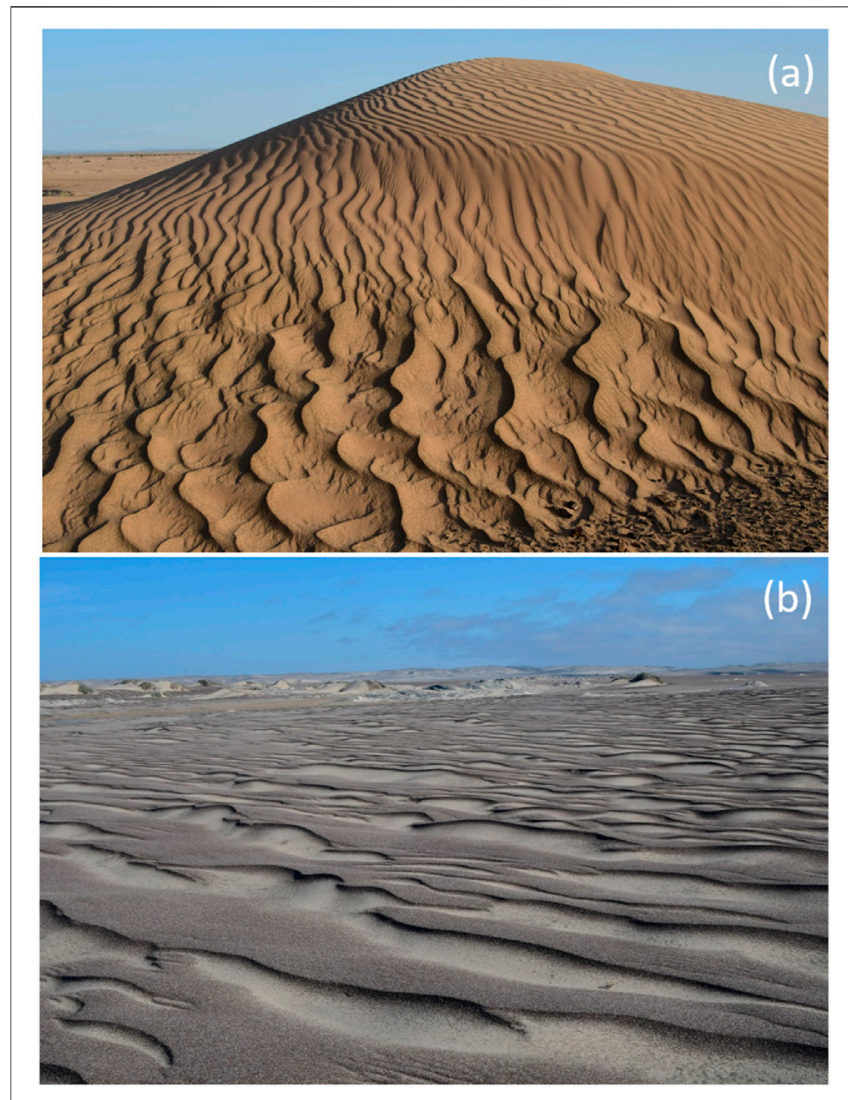

FIGURE 1 | (A) A transition between normal sand ripples at the top of a dune in Morocco near Merzouga to undulated megaripples at the bottom where coarse grains are more abundant. (B) A large field of megaripples near Torra Bay, Namibia. Notice the secondary small ripples superimposed on the windward slopes of the larger megaripples (both photos by Hezi Yizhaq).

time but did not saturate, whereas using bidisperse sand was found to stabilize the ripple growth due the emerging armoring layer with coarser particles, concentrated near the crests. This sorting effect due to the aeolian transport itself therefore seems to play an important role in the formation of both normal impact ripples and megaripples (Lämmel et al., 2018; Sullivan et al., 2020).

To better understand the growth instabilities causing bedform development, in the first place, requires a more accurate understanding of the shear-stress distribution in the flow over sand ripples. Such information can be obtained by CFD models (Siminovich et al., 2019; Yizhaq et al., 2021) and by low pressure wind tunnel measurements (Miller et al., 1987; Andreotti et al., 2021). Interestingly, in addition to the variations of the shear stress over undulated bedforms, also the basal pressure exhibits variations that break the symmetry and lag behind the topography (Claudin et al., in this special issue). The distribution of pressure on geophysical bedforms such as sand ripples is known to provide an important mechanism for accumulating moisture or dust within them (Louge et al., 2010). It will also become an important ingredient for a more complete quantitative description of ripple and megaripple formation.

\section{LARGE MARTIAN RIPPLES}

On Earth, ordinary impact ripple wavelengths are typically below $30 \mathrm{~cm}$, corresponding to crest heights less than $1 \mathrm{~cm}$, while megaripples can be considerably larger but are armored with coarse grains. However, on Mars, ripple-like bedforms can be small or large, both in wavelength and height, while their crests lack very coarse grains. It was argued that these two types of small and large ripples correspond to distinct size distributions, since intermediate transitional bedforms, roughly in the $20-80 \mathrm{~cm}$ range, seem to be lacking. Which could be indicative of two distinct formation mechanisms (Ewing et al., 2017). The large, meter-scale ripples without armoring layer have no corresponding terrestrial analog (Lapotre et al., 2016; Ewing et al., 2017; Vaz et al., 2017; Sullivan et al., 2020). They were detected in orbital images and first visited in situ by the NASA Mars Exploration Rover (MER) Spirit at the El Dorado ripple field in Gusev Crater (Sullivan et al., 2020). Based on data sent by the NASA Mars Science Laboratory (MSL) rover in Gale Crater a hypothesis for the origin of these meter scale ripples that superimpose dune surfaces at the MSL landing site was suggested (Lapotre et al., 2016; Ewing et al., 2017). According to this theory, the large ripples are fluid (or wind) drag ripples, which are similar in their morphology to subaqueous ripples. Recently, an interesting continuous transition between these enigmatic large ripples and megaripples has been observed on Mars by analyzing HiRISE images (Zimbelman, 2019), adding a new perspective to the ongoing debate on their formative mechanism (Duran Vinent et al., 2019; Lorenz, 2020; Sullivan et al., 2020; Lapotre et al., 2021; Yizhaq et al., 2021). It also remains unexplained why small ripples are found to be superimposed on the larger meter-scale ripples.

In any case, due to their large size, these ripples can be detected very well in orbital images and thus used to infer information about the wind flow over martian dunes (Hood et al., in this special issue). Hood et al. analyzed the ripple pattern and migration rate around dunes in the dune field of Nili Patera, which is one of the most active dune fields on Mars (Bridges et al., 2012). One of their findings is that changes in ripple patterns and migration rates in dune wakes indicate reattachment lengths for the atmospheric flow of 4-7 brink heights, consistent with the expected similarity of turbulent flow structure on Earth and Mars.

\section{TARs}

Yet another enigmatic bedform on Mars has been characterized as TARs (Transverse Aeolian Ridges), with no clear terrestrial counterpart (however, see Foroutan and Zimbelman, 2016; Foroutan et at., 2019). TARs are morphometrically similar to both ripples and dunes, with symmetric profiles like large wind ripples or small reversing dunes, and have wavelengths intermediate between ripples and dunes, somewhere on the 
order of 10-100 m. TARs seem to grow considerably larger than megaripples on Mars or Earth, but smaller than dunes on either planet, with amplitudes generally of a few to tens of meters. The interaction between large ripples and TARs as observed in Scandaia Cavi could be a new mechanism for TARs to grow (Fenton et al., in this special issue). By analyzing HiRISE images of the TARs in the wake of barchan dunes, (Fenton et al., in this special issue) moreover found TARs to interact with each other, exhibiting defect repulsions and possible lobe extensions, reminiscent of the coarsening of wind ripples. This is taken as an indication that these bedforms have migrated in the past.

It remains a challenge to arrive at a thorough understanding of the large Martian ripples and TARs and other aeolain bedforms, possibly still to be discovered in our solar system, in the future. To meet it, we will need a further and closer integration of theoretical

\section{REFERENCES}

Andreotti, B., Claudin, P., Iversen, J. J., Merrison, J. P., and Rasmussen, K. R. (2021). A lower-Than-expected Saltation Threshold at Martian Pressure and below. Proc. Natl. Acad. Sci. U S A. 118, 5-118. doi:10.1073/pnas.2012386118

Bridges, N. T., Ayoub, F., Avouac, J.-P., Leprince, S., Lucas, A., and Mattson, S. (2012). Earth-like Sand Fluxes on Mars. Nature 485 (7398), 339-342. doi:10.1038/nature11022

Day, M., and Zimbelman, J. (2021). Ripples, Megaripples, and TARs, Oh, My! Recommendations Regarding Mars Aeolian Bedform Terminology. Icarus 369, 114647. doi:10.1016/j.icarus.2021.114647

Duran Vinent, O., Andreotti, B., Claudin, P., and Winter, C. (2019). A Unified Model of Ripples and Dunes in Water and Planetary Environments. Nat. Geosci. 12 (5), 345-350. doi:10.1038/s41561-019-0336-4

Ewing, R. C., Lapotre, M. G. A., Lewis, K. W., Day, M., Stein, N., Rubin, D. M., et al. (2017). Sedimentary Processes of the Bagnold Dunes: Implications for the Eolian Rock Record of Mars. J. Geophys. Res. Planets 122 (12), 2544-2573. doi:10.1002/2017JE005324

Foroutan, M., Steinmetz, G., Zimbelman, J. R., and Duguay, C. R. (2019). Megaripples at Wau-An-Namus, Libya: A New Analog for Similar Features on Mars. Icarus 319, 840-851. doi:10.1016/j.icarus.2018.10.021

Foroutan, M., and Zimbelman, J. R. (2016). Mega-ripples in Iran: A New Analog for Transverse Aeolian Ridges on Mars. Icarus 274, 99-105. doi:10.1016/ j.icarus.2016.03.025

Fu, L.-T. (2021). Grain Size Ratio May Be Crucial for the Fluid Initiation of Martian Sand Grains. Icarus 358, 114225. doi:10.1016/j.icarus.2020.114225

Lämmel, M., Meiwald, A., Yizhaq, H., Tsoar, H., Katra, I., and Kroy, K. (2018). Aeolian Sand Sorting and Megaripple Formation. Nat. Phys 14, 759-765. doi:10.1038/s41567-018-0106-z

Lapôtre, M. G. A., Ewing, R. C., and Lamb, M. P. (2021). An Evolving Understanding of Enigmatic Large Ripples on Mars. J. Geophys. Res. Planets 126, e2020JE006729. doi:10.1029/2020JE006729

Lapotre, M. G. A., Ewing, R. C., Lamb, M. P., Fischer, W. W., Grotzinger, J. P., Rubin, D. M., et al. (2016). Large Wind Ripples on Mars: A Record of Atmospheric Evolution. Science 353, 55-58. doi:10.1126/science.aaf3206

Lorenz, R. D. (2020). Martian Ripples Making a Splash. J. Geophys. Res. Planets 125 (10), 12-15. doi:10.1029/2020je006658

Louge, M. Y., Valance, A., Mint Babah, H., Moreau-Trouvé, J-C., Ould el-Moctar, A., Dupont, P., et al. (2010). Seepage-induced Penetration of Water Vapor and Dust beneath Ripples and Dunes. J. Geophys. Res. 115, F02002. doi:10.1029/2009jf001385

Sennott-miller, L., Miller, J. L. L., and Greeley, R. (1987). Difficulty. Nurs. Res. 36, 268-271. doi:10.1097/00006199-198709000-00002 models, CFD simulations, and planetary wind tunnel experiments. Theory indicates that the unique atmospheric and granular conditions in various places of our solar system may possibly lead to new bedforms with no clear terrestrial analogs (Duran Vinent et al., 2019). They may in turn need new classification schemes and theories-but this is what makes this field of science so fascinating and will allow it to advance further, into possibly still unexpected directions.

\section{AUTHOR CONTRIBUTIONS}

All authors listed have made a substantial, direct, and intellectual contribution to the work and approved it for publication.

Silvestro, S., Chojnacki, M., Vaz, D. A., Cardinale, M., Yizhaq, H., and Esposito, F. (2020). Megaripple Migration on Mars. J. Geophys. Res. Planets 125 (8), e2020JE006446. doi:10.1029/2020JE006446

Siminovich, A., Elperin, T., Katra, I., Kok, J. F., Sullivan, R., Silvestro, S., et al. (2019). Numerical Study of Shear Stress Distribution over Sand Ripples under Terrestrial and Martian Conditions. J. Geophys. Res. Planets 124 (1), 175-185. doi:10.1029/2018je005701

Sullivan, R., Kok, J. F., Katra, I., and Yizhaq, H. (2020). A Broad Continuum of Aeolian Impact Ripple Morphologies on Mars Is Enabled by Low Wind Dynamic Pressures. J. Geophys. Res. Planets 125, 1-39. doi:10.1029/ 2020je006485

Tillman, N. (2021). Megaripples on Mars-How to Name Wind-Shaped Features on the Red Planet. Eos 102. doi:10.1029/2021EO162775

Vaz, D., Silvestro, S., Sarmento, P. T. K., and Cardinale, M. (2017). Migrating Meter-Scale Bedforms on Martian Dark Dunes: Are Terrestrial Aeolian Ripples Good Analogues. Aeolian Res. 26, 101-116. doi:10.1016/ j.aeolia.2016.08.003

Yizhaq, H., Bel, G., Silvestro, S., Elperin, T., Kok, J. F., Cardinale, M., et al. (2019). The Origin of the Transverse Instability of Aeolian Megaripples. Earth Planet. Sci. Lett. 512, 59-70. doi:10.1016/j.epsl.2019.01.025

Yizhaq, H., Siminovich, A., Katra, I., Levi, A., Sullivan, R., Silvestro, S., et al. (2021). Turbulent Shear Flow over Large Martian Ripples. J. Geophys. Res. - Planets 126. doi:10.1029/2020JE006515

Zimbelman, J. R. (2019). The Transition between Sand Ripples and Megaripples on Mars. Icarus 333, 127-129. doi:10.1016/j.icarus.2019.05.017

Conflict of Interest: The authors declare that the research was conducted in the absence of any commercial or financial relationships that could be construed as a potential conflict of interest.

Publisher's Note: All claims expressed in this article are solely those of the authors and do not necessarily represent those of their affiliated organizations, or those of the publisher, the editors and the reviewers. Any product that may be evaluated in this article, or claim that may be made by its manufacturer, is not guaranteed or endorsed by the publisher.

Copyright (c) 2021 Yizhaq, Silvestro and Kroy. This is an open-access article distributed under the terms of the Creative Commons Attribution License (CC $B Y)$. The use, distribution or reproduction in other forums is permitted, provided the original author(s) and the copyright owner(s) are credited and that the original publication in this journal is cited, in accordance with accepted academic practice. No use, distribution or reproduction is permitted which does not comply with these terms. 